\title{
DESIGUALDAD DE GÉNERO EN TIEMPOS DE CRISIS: ANÁLISIS COMPARATIVO DE LA ECONOMÍA SOCIAL
}

\author{
POR \\ $\mathrm{M}^{\mathrm{a}}$ Antonia RIBAS BONET* $\mathrm{y}$ \\ Antonia SAJARDO MORENO**
}

\section{RESUMEN:}

La crisis económica en la que aún se halla inmersa la economía española ha sido especialmente procaz en el mercado laboral. Las abultadas cifras de desempleo parecen enmascarar una tétrica situación que es experimentada de desigual forma por hombres y mujeres. En este contexto, el objetivo fundamental de este artículo estriba en analizar si las diferencias de género existentes en el mercado laboral español se manifiestan del mismo modo durante la actual crisis o bien se aprecian diferencias entre la época de recesión y la inmediatamente anterior. Valorar si la crisis ha afectado por igual a los miembros de uno y otro sexo o si ha habido diferencias sustanciales entre ellos, conforma el hilo conductor de este trabajo, y en su seno evidenciar las eventuales diferencias existentes entre las empresas de Economía Social y el resto de empresas de la economía española.

Palabras clave: desigualdad de género, crisis económica, economía social

Claves ECONLIT: J160, J710, L300, L310, L390

\footnotetext{
* Departamento de Economía Aplicada. UNIVERSITAT DE LES ILLES BALEARS. Dirección de correo electrónico: marian.ribas@ uib.es

** Departamento de Economía Aplicada. UNIVERSITAT DE VALÉNCIA: Dirección de correo electrónico: sajardo@uv.es
}

REVESCO No 105 - MONOGRÁFICO: La economía social y la igualdad de género -

ISSN: 1885-8031 - www.ucm.es/info/revesco

DOI: $10.5209 /$ rev_REVE.2011.v105.2

Fecha de recepción: 18/11/2010

Fecha de aceptación: 05/04/2011 


\title{
GENDER INEQUALITY IN TIMES OF CRISIS: A COMPARATIVE ANALYSIS OF SOCIAL ECONOMY
}

\begin{abstract}
:
The economic crisis which is still engaged the Spanish economy has been especially brazen in the labor market. The bulging unemployment figures seem to mask a grim situation that is experienced unequally by men and women form. In this context, the primary objective of this paper is to examine whether gender differences exist in the Spanish labor market is expressed in the same way during the current crisis or differences were found between the time of the preceding recession. Assess whether the crisis has affected equally to members of either sex or if there are substantial differences between them, forms the theme of this work, and within it reveal any differences between the companies and the Social Economy other companies of the Spanish economy.
\end{abstract}

Keywords: gender inequality, economic crisis, social economy

\section{INTRODUCCIÓN}

El interés fundamental de este trabajo es la escasez de estudios que aborden la cuestión del género en el ámbito de la Economía Social, y más aún analizando el tema a lo largo de una época recesiva en la que el empleo se ve afectado significativamente. No obstante, estudios anteriores ya han puesto de manifiesto la existencia de desigualdades de género en el sector de la Economía Social. El Ministerio de Trabajo e Inmigración desde hace ya varios años publica datos relativos a las entidades de dicho sector desagregados por sexo. Por su parte, la Confederación Española de Cooperativas de Trabajo Asociado ha sido una de las organizaciones pioneras en la realización de estudios sobre la situación de la mujer en el ámbito cooperativo y en la promoción de publicaciones e iniciativas que puedan ayudar a la visibilidad de la problemática del colectivo femenino dentro el movimiento cooperativo.

Otros trabajos (Senent, 2008) analizan las desigualdades de género en el marco de la Economía Social desde un punto de vista teórico, partiendo de los postulados que rigen las empresas cooperativas (principios cooperativos) y analizando la discordancia existente entre 
la situación de las mujeres en las cooperativas y las premisas teóricas que les son aplicables. Quizá los estudios de Ribas (2005 y 2010) y los trabajos de Ribas y Sajardo (2004 y 2005) son los que abordan en mayor profundidad la cuestión del género en el ámbito de la Economía Social de una forma global, ya que se analiza el tema en las diversas entidades que componen el sector, y además de forma exhaustiva, dado que se acomete el estudio de las distintas variables relacionadas con el mercado laboral: Porcentajes de participación globales, por sectores, ramas de actividad...; tipología de contratos; jornada laboral, antigüedad, etc.

Las conclusiones de dichos trabajos destacan la existencia inequívoca de importantes desigualdades de género en un sector que presenta características específicas que podrían ser determinantes en la consecución de una mayor equidad entre los miembros de uno y otro sexo. Es por ello, que el presente trabajo retoma dicho ámbito de estudio para realizar un análisis de las principales variables del mercado de trabajo en una época marcada por la crisis económica que ha determinado un importante descenso del empleo. El objetivo es lograr una comparación entre las cifras relativas al sector de la Economía Social y el conjunto de la economía española, al mismo tiempo que se pretende una visión temporal que permita observar la evolución de los datos de empleo desde la época anterior a la crisis hasta llegar al momento en que ésta se ha presentado de un modo más acusado.

La época de crisis que se ha desencadenado en los últimos dos años ha convulsionado el mercado laboral. Una de las consecuencias más visibles de la crisis ha sido el importante incremento de la tasa de paro y del número de desempleados. En este contexto, es evidente que la precaria situación laboral ha afectado a hombres y mujeres, sin embargo, la cuestión clave que se puede plantear es si la crisis ha afectado por igual a los miembros de uno y otro sexo o si ha habido diferencias sustanciales entre ellos. Ese es el tema objeto de análisis de este trabajo. El objetivo fundamental de este artículo es discernir si las diferencias de género existentes en el mercado laboral español se manifiestan del mismo modo durante la época de crisis o bien se aprecian diferencias entre la época de recesión y la inmediatamente anterior.

En el contexto de crisis ya mencionado, interesa asimismo, evidenciar las eventuales diferencias entre las empresas de Economía Social y el resto de empresas de la economía española. Para lograr dicho objetivo se estudian de forma separada los datos de la Encuesta de Población Activa publicados por el Instituto Nacional de Estadística (INE) y las estadísticas 
de la base de datos del Ministerio de Trabajo e Inmigración relativos a las empresas de Economía Social (cooperativas, sociedades laborales anónimas y sociedades anónimas limitadas) estableciendo una comparación entre ambas fuentes.

Para lograr dichos objetivos a lo largo de la exposición se analizarán diversas variables relativas al mercado laboral español en función del sexo como son las tasas de paro y de actividad, la población activa, ocupada y parada, los diferentes sectores de actividad, los tipos de contrato y el tipo de jornada laboral. Para el análisis se toma una franja temporal que comprende desde el primer trimestre del 2007 (antes del comienzo de la época de crisis) hasta la actualidad (segundo trimestre del 2010). Los datos son presentados por trimestres con el objeto de facilitar una percepción más clara de la evolución de las cifras a lo largo del período.

\section{COMPARACIÓN DE LA PARTICIPACIÓN LABORAL DE HOMBRES Y MUJERES}

En este primer epígrafe, y como punto inicial a abordar en el presente trabajo, se analiza la participación laboral de hombres y mujeres a dos niveles: por un lado en relación al conjunto del mercado de trabajo español y por otro lado centrando el estudio en las entidades de Economía Social (concretamente cooperativas y sociedades laborales). De este modo se obtiene una perspectiva comparativa entre el comportamiento del mercado laboral español y el que presentan las entidades de economía social en relación a la cuestión del género.

Seguidamente, en la tabla 1 se presentan datos sobre las tasas de paro y actividad en el conjunto de la economía española durante los años 2007 al 2010, inclusive, lo que nos da una visión de la evolución de las mismas.

Tabla 1: Tasas de paro y de actividad según sexo. ${ }^{1}$

\begin{tabular}{|c|c|c|c|c|}
\hline \multirow{2}{*}{ año } & \multicolumn{2}{|c|}{ Tasas de paro (\%) } & \multicolumn{2}{c|}{ Tasas de actividad (\%) } \\
\cline { 2 - 5 } & Hombres* & Mujeres* & Hombres & Mujeres \\
\hline
\end{tabular}

1 La tasa de paro se define como el cociente entre el número de personas en paro y la población activa. La tasa de actividad se obtiene dividiendo el número de personas activas entre la población de dieciséis o más años.

REVESCO No 105 - MONOGRÁFICO: La economía social y la igualdad de género -

ISSN: 1885-8031 - www.ucm.es/info/revesco 


\begin{tabular}{|l|l|l|l|l|}
\hline 1T 2007 & 6,3 & 11,4 & 68,9 & 48,6 \\
\hline 2T 2007 & 6,1 & 10,5 & 69,3 & 48,8 \\
\hline 3T 2007 & 6,2 & 10,5 & 69,6 & 49,0 \\
\hline 4T 2007 & 6,8 & 11,0 & 69,2 & 49,4 \\
\hline 1T 2008 & 7,9 & 12,0 & 69,3 & 49,7 \\
\hline 2T 2008 & 9,1 & 12,3 & 69,6 & 50,3 \\
\hline 3T 2008 & 10,3 & 12,7 & 69,8 & 50,5 \\
\hline 4T 2008 & 13,0 & 15,1 & 69,2 & 51,4 \\
\hline 1T 2009 & 16,9 & 18,0 & 69,1 & 51,5 \\
\hline 2T 2009 & 17,6 & 18,3 & 68,9 & 51,6 \\
\hline 3T 2009 & 17,8 & 18,2 & 68,4 & 51,5 \\
\hline 4T 2009 & 18,6 & 19,1 & 68,1 & 51,7 \\
\hline 1T 2010 & 20,0 & 20,2 & 68,0 & 52,1 \\
\hline 2T 2010 & 19,7 & 20,6 & 68,4 & 52,2 \\
\hline
\end{tabular}

Fuente: Encuesta de Población Activa, Instituto Nacional de Estadística, Ministerio de Economía y Hacienda (http://www.ine.es/)

* En adelante $H$ y $M$

Siguiendo los datos estadísticos del INE se puede constatar que durante el período que comprende la época de crisis aguda, concretamente el periodo comprendido entre comienzos del 2007 y el segundo trimestre del 2010, se observa que la evolución de las tasas de paro y las tasas de actividad en hombres y mujeres presentan evoluciones distintas.

Al comparar las tasas de paro registradas entre la población masculina y la femenina se pone de manifiesto que los hombres han acusado la situación de crisis en mayor medida que las mujeres. La tasa de paro entre los varones se ha visto incrementada considerablemente, llegando a triplicarse entre el primer trimestre del 2007 y el segundo trimestre del 2010, concretamente dicha tasa ha pasado del 6,3\% al 19,7 \% en el mencionado período. En el caso de las mujeres la tasa de paro en el segundo trimestre del 2010 alcanza el 20,56 \% (cifra bastante cercana a la registrada entre el colectivo masculino aunque algo más elevada), sin embargo hay que tomar en consideración el hecho de que históricamente las mujeres han venido presentando tasas de paro más elevadas y que en el primer trimestre del 2007 la tasa de paro femenino se situaba en el 11,4\% (cerca del doble de la tasa de paro 
masculino). Estos datos revelan que aunque hombres y mujeres se han visto afectados por la situación de crisis económica, los hombres han sufrido sus consecuencias en mayor medida que las mujeres.

Al analizar las tasas de actividad de los miembros de uno y otro sexo destaca el hecho de que la tasa de actividad entre los hombres se ha mantenido constante a lo largo del período estudiado sufriendo únicamente pequeñas oscilaciones y situándose en un intervalo comprendido entre un mínimo del $68 \%$ (primer trimestre de 2010) y un máximo del 69,6\% registrado el tercer trimestre del 2008. Por el contrario, la evolución de la tasa de actividad del colectivo femenino es significativamente distinta, puesto que presenta una tendencia claramente ascendente incrementándose desde el 48,6 \% registrado el primer trimestre del 2007 hasta el 52,2 \% registrado durante el segundo trimestre del 2010, lo cual constata una mayor incorporación laboral de las mujeres durante el periodo analizado que se traduce en un incremento de alrededor de cuatro puntos porcentuales en la tasa de actividad femenina.

Tabla 2: Distribución porcentual de la población activa, ocupada y parada según sexo. ${ }^{2}$

\begin{tabular}{|c|c|c|c|c|c|c|}
\hline \multirow{2}{*}{ Año } & \multicolumn{2}{|c|}{ Activos } & \multicolumn{2}{c|}{ Ocupados } & \multicolumn{2}{c|}{ Parados } \\
\cline { 2 - 7 } & \% H & \% M & \% H & \% M & \% H & \% M \\
\hline 1T 2007 & 57,7 & 42,3 & 59,1 & 40,9 & 43,1 & 56,9 \\
\hline 2T 2007 & 57,8 & 42,2 & 59,0 & 41,0 & 44,4 & 55,6 \\
\hline 3T 2007 & 57,8 & 42,2 & 58,9 & 41,1 & 44,7 & 55,3 \\
\hline 4T 2007 & 57,5 & 42,5 & 58,6 & 41,4 & 45,6 & 54,4 \\
\hline 1T 2008 & 57,3 & 42,7 & 58,4 & 41,6 & 46,8 & 53,2 \\
\hline
\end{tabular}

2 El INE define dichas categorías tal y como sigue:

- Población activa: "personas de 16 o más años que, durante la semana de referencia (la anterior a aquella en que se realiza la entrevista), suministran mano de obra para la producción de bienes y servicios o están disponibles y en condiciones de incorporarse a dicha producción. Se subdividen en ocupados y parados."

- Población ocupada: "personas de 16 o más años que durante la semana de referencia han estado trabajando durante al menos una hora, a cambio de una retribución (salario, jornal, beneficio empresarial,...) en dinero o especie. También son ocupados quienes teniendo trabajo han estado temporalmente ausentes del mismo por enfermedad, vacaciones, etcétera."

- Población en paro: "personas de 16 o más años que durante la semana de referencia han estado sin trabajo, disponibles para trabajar y buscando activamente empleo. Son parados también quienes ya han encontrado un trabajo y están a la espera de incorporarse a él, siempre que verifiquen las dos primeras condiciones."

REVESCO No 105 - MONOGRÁFICO: La economía social y la igualdad de género -

ISSN: 1885-8031 - www.ucm.es/info/revesco 


\begin{tabular}{|c|c|c|c|c|c|c|}
\hline 2T 2008 & 57,2 & 42,8 & 58,1 & 41,9 & 49,7 & 50,3 \\
\hline 3T 2008 & 57,1 & 42,9 & 57,8 & 42,2 & 52,1 & 47,9 \\
\hline 4T 2008 & 56,5 & 43,5 & 57,1 & 42,9 & 52,6 & 47,4 \\
\hline 1T 2009 & 56,4 & 43,6 & 56,7 & 43,3 & 54,7 & 45,3 \\
\hline 2T 2009 & 56,3 & 43,7 & 56,5 & 43,5 & 55,3 & 44,7 \\
\hline 3T 2009 & 56,1 & 43,9 & 56,2 & 43,8 & 55,6 & 44,4 \\
\hline 4T 2009 & 55,9 & 44,1 & 56,0 & 44,0 & 55,3 & 44,7 \\
\hline 1T 2010 & 55,6 & 44,4 & 55,7 & 44,3 & 55,4 & 44,6 \\
\hline 2T 2010 & 55,7 & 44,3 & 55,9 & 44,1 & 54,6 & 45,4 \\
\hline
\end{tabular}

Fuente: Encuesta de Población Activa, Instituto Nacional de Estadística, Ministerio de Economía y Hacienda (http://www.ine.es/) y elaboración propia.

Al estudiar la distribución según sexo de la población activa, ocupada y parada también aparecen diferencias significativas en la evolución del peso de hombres y mujeres dentro de cada uno de los colectivos mencionados. Durante el periodo analizado (primer trimestre del 2007 y segundo trimestre del 2010) se observa un incremento de dos puntos porcentuales del peso de las mujeres dentro de la población activa que pasan de representar un $42,3 \%$ a suponer un $44,3 \%$. La contrapartida de dicho incremento es un decremento de dos puntos porcentuales en el peso de los hombres entre la población activa cuyo porcentaje de participación pasa de un $57,7 \%$ a un $55,7 \%$.

Las cifras relativas a la distribución de hombres y mujeres entre la población ocupada ofrecen un resultado similar al ya comentado para la población activa. A lo largo del período analizado se produce un descenso de tres puntos porcentuales en la proporción de hombres (desde un 59,1\% a un 55,9\%) al mismo tiempo que se observa un incremento equivalente en el peso de las mujeres entre la población ocupada, que pasa de un 40,9\% a un 44,1\%.

La evolución de la distribución por sexo del colectivo de parados presenta una evolución opuesta a la observada en los colectivos de activos y ocupados, y una tendencia concordante con la evolución de las tasas de paro comentadas anteriormente. Así, encontramos que el peso de los hombres entre el colectivo de parados/as se ve incrementado en más de diez puntos porcentuales pasando de un $43,1 \%$ a un $54,6 \%$, mientras que la proporción de mujeres presenta la tendencia contraria descendiendo considerablemente y 
pasando de un 56,9 \% en el primer trimestre del 2007 a un 45,4\% durante el segundo trimestre del 2010. Estos datos confirman una mejor tendencia laboral para las mujeres en relación a la experimentada por los hombres quienes presentan un mayor retroceso en su presencia en el mercado de trabajo durante la época de crisis. Una explicación a esta evolución vendría dada por el hecho de que uno de los sectores más afectados por la recesión económica y que ha causado un porcentaje importante de desempleo ha sido en particular el sector de la construcción ampliamente masculinizado, lo cual contribuiría en cierto modo a que los hombres vean incrementado su peso entre el grupo de parados en favor de una mayor proporción de mujeres.

Una vez expuestas las cifras relativas al conjunto del mercado de trabajo español resulta de interés comparar los datos de participación laboral a nivel general con la proporción de hombres y mujeres que se registran en las entidades de Economía Social y que se presentan a continuación.

Tabla 3: Distribución porcentual de los trabajadores en las Sociedades de Economía Social, según sexo.

\begin{tabular}{|c|c|c|c|c|c|c|c|c|}
\hline \multirow{2}{*}{ Añ } & \multicolumn{2}{|c|}{$\begin{array}{c}\text { Total } \\
\text { economía }\end{array}$} & \multicolumn{2}{|c|}{$\begin{array}{c}\text { sooperativas } \\
\text { social }\end{array}$} & \multicolumn{2}{c|}{$\begin{array}{c}\text { Soc. lab. } \\
\text { anonimas }\end{array}$} & \multicolumn{2}{c|}{$\begin{array}{c}\text { Soc. lab. } \\
\text { limitadas }\end{array}$} \\
\cline { 2 - 9 } & \% H & \% M & \% H & \% M & \% H & M & \% H & \% M \\
\hline 1T 2007 & 59,2 & 40,8 & 54,3 & 45,7 & 72,5 & 27,5 & 70,4 & 29,6 \\
\hline 2T 2007 & 61,1 & 38,9 & 56,4 & 43,6 & 74,1 & 25,9 & 70,8 & 29,2 \\
\hline 3T 2007 & 60,9 & 39,1 & 56,4 & 43,6 & 73,7 & 26,3 & 70,4 & 29,6 \\
\hline 4T 2007 & 59,2 & 40,8 & 55,0 & 45,0 & 72,7 & 27,3 & 68,8 & 31,2 \\
\hline 1T 2008 & 58,1 & 41,9 & 53,5 & 46,5 & 72,1 & 27,9 & 68,9 & 31,1 \\
\hline 2T 2008 & 59,6 & 40,4 & 55,5 & 44,5 & 72,3 & 27,7 & 68,7 & 31,3 \\
\hline 3T 2008 & 59,0 & 41,0 & 55,3 & 44,7 & 72,0 & 28,0 & 67,6 & 32,4 \\
\hline 4T 2008 & 57,2 & 42,8 & 54,1 & 45,9 & 71,1 & 28,9 & 65,1 & 34,9 \\
\hline 1T 2009 & 55,9 & 44,1 & 52,3 & 47,7 & 71,3 & 28,7 & 65,0 & 35,0 \\
\hline 2T 2009 & 57,4 & 42,6 & 54,1 & 45,9 & 71,2 & 28,8 & 65,6 & 34,4 \\
\hline 3T 2009 & 57,4 & 42,6 & 54,4 & 45,6 & 70,7 & 29,3 & 65,0 & 35,0 \\
\hline 4T 2009 & 55,9 & 44,1 & 53,2 & 46,8 & 69,6 & 30,4 & 63,1 & 36,9 \\
\hline
\end{tabular}

REVESCO Nº 105 - MONOGRÁFICO: La economía social y la igualdad de género -

ISSN: 1885-8031 - www.ucm.es/info/revesco 


\begin{tabular}{|l|l|l|l|l|l|l|l|l|}
\hline 1T 2010 & 55,3 & 44,7 & 52,3 & 47,7 & 69,2 & 30,8 & 63,4 & 36,6 \\
\hline 2T 2010 & 56,6 & 43,4 & 53,6 & 46,4 & 69,2 & 30,8 & 64,7 & 35,3 \\
\hline
\end{tabular}

Fuente: Ministerio de Trabajo e inmigración. Dirección General de la Economía Social, del trabajo autónomo y de la responsabilidad social de las empresas (http://www.mtin.es).

El análisis de la distribución por sexo de los trabajadores de la Economía Social muestra una tendencia semejante a la observada para el conjunto de la economía española. Tal como se puede apreciar en la tabla 3, durante el período analizado se observa una tendencia descendente en la proporción de hombres entre el colectivo de trabajadores de la Economía Social (de un 59,2 \% a un 56,6 \%) a la vez que se registra un incremento en el porcentaje de mujeres en dicho colectivo que pasan de representar un 40,8 \% a poseer una representación del 43,4 \% en el segundo trimestre del 2010. Autoras como Ribas y Sajardo (2004), Senent (2008) o Ribas (2010), hacen hincapié en las diferencias de género existentes en el ámbito de la Economía Social a pesar de los principios que conforman dicho sector, representando, por tanto, una incongruencia con los valores propios de las entidades de Economía Social.

No obstante lo anterior, cabe destacar que no todas las empresas de la Economía Social (cooperativas, sociedades laborales anónimas y sociedades laborales limitadas) presentan datos similares. La mayor participación femenina se registra en las cooperativas y se mantiene bastante estable a lo largo del período objeto de estudio, mostrando oscilaciones dentro de un intervalo de dos puntos porcentuales y presentando cifras más parecidas a las registradas para los colectivos de población activa y población ocupada en España. Las autoras Ribas y Sajardo (2005) han puesto de manifiesto anteriormente las diferencias de las distintas organizaciones de la Economía Social en relación a la igualdad de género y resaltando las preferencias de las mujeres en relación a su participación en las distintas entidades integradas en dicho sector.

En comparación a los datos registrados en las cooperativas, el peso femenino entre los trabajadores de las sociedades laborales es significativamente inferior, especialmente en el caso de las sociedades laborales anónimas en las cuales la presencia femenina en el segundo trimestre del 2010 únicamente llega a alcanzar un 30,8 \%, y ello a pesar de haberse incrementado más de tres puntos porcentuales desde el primer trimestre del 2007, fecha en la que las mujeres tan sólo representan un porcentaje del 27,5 \%. En las sociedades laborales

REVESCO Nº 105 - MONOGRÁFICO: La economía social y la igualdad de género -

ISSN: 1885-8031 - www.ucm.es/info/revesco 
limitadas el peso del colectivo se incrementa desde un 29,6\% hasta alcanzar un 35,3\% al final del período analizado, en perjuicio de la representación masculina que se ve mermada en cerca de seis puntos porcentuales. No obstante lo anterior, los datos observados en las sociedades laborales limitadas, aun siendo significativamente superiores a los de las sociedades laborales anónimas son muy inferiores a los observados en el sector de la Economía Social, en las cooperativas y en el conjunto del mercado laboral español. Sin embargo, a pesar de dicha evidencia empírica, las cifras confirman una tendencia semejante en las entidades de la Economía Social y en el conjunto de la economía española, que no es otra que el constante avance de la presencia femenina en detrimento del peso del colectivo masculino y, por tanto, del logro de estadísticas más igualitarias (menos desequilibradas) para hombres y mujeres.

Las estadísticas de miembros de cooperativas arrojados por la Encuesta de Población Activa (Instituto Nacional de Estadística: http://www.ine.es/) muestran resultados distintos a los publicados en la base de datos del Ministerio de Trabajo e Inmigración (Dirección General de la Economía Social, del trabajo autónomo y de la responsabilidad social de las empresas: http://www.mtin.es) elaborada sobre datos reales de cotización de las empresas de Economía Social, ello es debido a que estos últimos incluyen los trabajadores de la empresa y no únicamente los socios/as de la cooperativa. Las cifras del Instituto Nacional de Estadística muestran que el porcentaje de mujeres se ha venido incrementando en las cooperativas a lo largo del período analizado, pasando desde el 29,8\% en el primer trimestre del 2007 hasta el $40,3 \%$ en el segundo trimestre del 2010, siendo estos porcentajes significativamente inferiores a los mostrados por la base de datos de la Economía Social para el conjunto de los trabajadores de las cooperativas.

\section{COMPARACIÓN DE LA PARTICIPACIÓN HOMBRES Y MUJERES SEGÚN SECTORES DE ACTIVIDAD}

Tal y como se pone de relieve en los numerosos estudios sobre género, uno de los rasgos más significativos en relación a la discriminación entre los miembros de uno y otro sexo es la desigual presencia de hombres y mujeres en los distintos sectores de actividad

REVESCO No 105 - MONOGRÁFICO: La economía social y la igualdad de género -

ISSN: 1885-8031 - www.ucm.es/info/revesco 
(segregación horizontal), hecho que, tal y como se observa en los datos presentados en las tablas 4 y 5 , se manifiesta tanto a nivel general como en las empresas de Economía Social.

Tabla 4: Distribución porcentual de la población ocupada por sector de actividad, según sexo*.

\begin{tabular}{|c|c|c|c|c|c|c|c|c|}
\hline \multirow{2}{*}{ AÑ } & \multicolumn{2}{|c|}{ Agricultura } & \multicolumn{2}{|c|}{ Industria } & \multicolumn{2}{c|}{ Construcción } & \multicolumn{2}{c|}{ Servicios } \\
\cline { 2 - 10 } & \% H & \% M & \% H & \% M & \% H & \% M & \% H & \% M \\
\hline 1T 2008 & 72,0 & 28,0 & 75,0 & 25,0 & 93,3 & 6,7 & 46,7 & 53,3 \\
\hline 2T 2008 & 71,9 & 28,1 & 75,7 & 24,3 & 93,0 & 7,0 & 46,6 & 53,4 \\
\hline 3T 2008 & 74,6 & 25,4 & 75,7 & 24,3 & 92,9 & 7,1 & 46,7 & 53,3 \\
\hline 4T 2008 & 73,6 & 26,4 & 76,0 & 24,0 & 92,6 & 7,4 & 46,4 & 53,6 \\
\hline 1T 2009 & 72,8 & 27,2 & 76,1 & 24,0 & 92,1 & 7,9 & 46,3 & 53,7 \\
\hline 2T 2009 & 74,2 & 25,8 & 75,7 & 24,3 & 92,6 & 7,4 & 46,3 & 53,7 \\
\hline 3T 2009 & 75,3 & 24,7 & 75,2 & 24,8 & 92,6 & 7,4 & 46,4 & 53,6 \\
\hline 4T 2009 & 73,7 & 26,3 & 74,8 & 25,2 & 92,1 & 7,9 & 46,3 & 53,7 \\
\hline 1T 2010 & 71,9 & 28,1 & 75,4 & 24,6 & 91,0 & 9,0 & 46,4 & 53,6 \\
\hline 2T 2010 & 74,4 & 25,6 & 75,5 & 24,5 & 91,6 & 8,4 & 46,5 & 53,5 \\
\hline
\end{tabular}

* Datos disponibles desde el primer trimestre de 2008.

Fuente: Encuesta de Población Activa, Instituto Nacional de Estadística, Ministerio de Economía y Hacienda (http://www.ine.es/) y elaboración propia.

Es especialmente evidente la existencia de segregación laboral horizontal en el mercado laboral español. Hombres y mujeres participan de forma desigual en los distintos sectores de actividad, algunos de ellos están masculinizados y en otros casos como es el del sector servicios se observa una mayor presencia femenina.

En el sector de la agricultura tan sólo una cuarta parte de los trabajadores son mujeres. La presencia del colectivo femenino oscila cíclicamente en función del trimestre, es decir, según la estación del año, y por tanto, de las necesidades propias de la actividad agrícola en cada período. De este modo se observa que en los primeros trimestres del año, especialmente el primero, existe una mayor proporción de mujeres llegando a sobrepasar el $28 \%$, para descender en la segunda mitad del año.

REVESCO Nº 105 - MONOGRÁFICO: La economía social y la igualdad de género -

ISSN: 1885-8031 - www.ucm.es/info/revesco 
A diferencia del sector de la agricultura los restantes sectores presentan una distribución de hombres y mujeres bastante estable. En la industria la presencia femenina se sitúa en torno al $24 \%$ ó $25 \%$ sin apenas variaciones durante los trimestres analizados. Lo mismo ocurre en el sector servicios, en el que las mujeres representan más del $53 \%$ de los trabajadores, cifra que se mantiene constante desde principios del 2008 hasta la actualidad.

El colectivo femenino posee una participación residual en el sector de la construcción, sin embargo, se detecta que la proporción de mujeres en el sector presenta una tendencia moderadamente alcista, habiéndose incrementado desde el 6,7 \% en el primer trimestre del 2008 hasta el 8,4\% que se registra en el segundo trimestre del 2010. Una explicación a este dato podría ser el hecho de que los puestos que ocupan las mujeres en el sector de la construcción son fundamentalmente cargos técnicos y administrativos, quizá afectados en menor media por la crisis que los trabajos mayoritariamente realizados por los varones a pie de obra.

Tabla 5: Distribución porcentual de los trabajadores de la Economía Social por sector de actividad, según sexo.

\begin{tabular}{|c|c|c|c|c|c|c|c|c|}
\hline \multirow{2}{*}{ AÑ } & \multicolumn{2}{|c|}{ Agricultura } & \multicolumn{2}{|c|}{ Industria } & \multicolumn{2}{c|}{ Construcción } & \multicolumn{2}{c|}{ Servicios } \\
\cline { 2 - 9 } & \% H & \% M & \% H & \% M & \% H & \% M & \% H & \% M \\
\hline 1T 2007 & 48,9 & 51,1 & 73,0 & 27,0 & 93,9 & 6,1 & 46,4 & 53,6 \\
\hline 2T 2007 & 53,7 & 46,3 & 73,2 & 26,8 & 93,9 & 6,1 & 48,1 & 51,9 \\
\hline 3T 2007 & 59,4 & 40,6 & 73,2 & 26,8 & 93,7 & 6,3 & 47,3 & 52,7 \\
\hline 4T 2007 & 61,0 & 39,0 & 73,6 & 26,4 & 93,4 & 6,6 & 45,2 & 54,8 \\
\hline 1T 2008 & 47,3 & 52,7 & 73,4 & 26,6 & 93,5 & 6,5 & 45,5 & 54,5 \\
\hline 2T 2008 & 55,2 & 44,8 & 73,5 & 26,5 & 93,3 & 6,7 & 46,9 & 53,1 \\
\hline 3T 2008 & 61,2 & 38,8 & 73,7 & 26,3 & 92,9 & 7,1 & 46,0 & 54,0 \\
\hline 4T 2008 & 63,3 & 36,7 & 74,1 & 25,9 & 92,2 & 7,8 & 44,3 & 55,7 \\
\hline 1T 2009 & 48,8 & 51,2 & 73,9 & 26,1 & 92,0 & 8,0 & 44,7 & 55,3 \\
\hline 2T 2009 & 55,1 & 44,9 & 74,0 & 26,0 & 92,4 & 7,6 & 45,9 & 54,1 \\
\hline 3T 2009 & 60,8 & 39,2 & 73,9 & 26,1 & 92,3 & 7,7 & 45,4 & 54,6 \\
\hline 4T 2009 & 64,5 & 35,5 & 74,3 & 25,7 & 91,7 & 8,3 & 43,7 & 56,3 \\
\hline 1T 2010 & 51,8 & 48,2 & 74,2 & 25,8 & 92,0 & 8,0 & 44,1 & 55,9 \\
\hline
\end{tabular}

REVESCO No 105 - MONOGRÁFICO: La economía social y la igualdad de género -

ISSN: 1885-8031 - www.ucm.es/info/revesco 


\begin{tabular}{|l|l|l|l|l|l|l|l|l|}
\hline 2T 2010 & 56,9 & 43,1 & 74,3 & 25,7 & 92,4 & 7,6 & 45,2 & 54,8 \\
\hline
\end{tabular}

Fuente: Ministerio de Trabajo e inmigración. Dirección General de la Economía Social, del trabajo autónomo y de la responsabilidad social de las empresas (http://www.mtin.es).

Al analizar la distribución por sexo de los trabajadores de las empresas de Economía Social se observan algunas diferencias en relación a los resultados observados para el conjunto de la población española. El dato más destacable es la mayor participación femenina en el sector de la agricultura. El peso de hombres y mujeres en el sector de la Economía Social presenta una tendencia cíclica oscilante igual que ocurre para el conjunto de la economía, sin embargo la presencia del colectivo femenino supera en más de 20 puntos porcentuales las cifras arrojadas por el INE para la economía en general. De este modo en el sector de la Economía Social las mujeres llegan a alcanzar una mayoría de hasta un 52,7 \% en el primer trimestre del 2008.

En el sector de la industria la proporción de hombres y mujeres se mantiene estable aunque con una leve tendencia a la baja en el caso del colectivo femenino que pasa de un 27 $\%$ en el primer trimestre del 2007 a un 25,7 \% en el segundo trimestre del 2010. Las estadísticas registradas en el sector de la Economía Social son semejantes, por tanto, a las obtenidas para el conjunto de la economía. Una situación similar se observa en el sector de la construcción. Las cifras relativas a la Economía Social son equivalentes a las registradas a nivel general incrementándose la proporción de mujeres desde un $6,1 \%$ hasta el 7,6\% durante el periodo analizado.

El colectivo femenino en el sector servicios de la Economía Social presenta datos similares a los ofrecidos por el INE a nivel general con una mayoría de mujeres, no obstante, en las entidades del tercer sector se observa un comportamiento cíclico y una tendencia levemente alcista llegando incluso a alcanzar un 56,3\% en el cuarto trimestre del 2009.

REVESCO Nº 105 - MONOGRÁFICO: La economía social y la igualdad de género -

ISSN: 1885-8031 - www.ucm.es/info/revesco 


\section{COMPARACIÓN DEL TIPO DE RELACIÓN LABORAL DE HOMBRES Y MUJERES}

Además de observar diferencias de participación en el mercado de trabajo y distinta presencia según sector de actividad de los miembros de uno y otro sexo, la evidencia empírica muestra que los hombres y mujeres integrados en el mercado laboral, no desarrollan su actividad bajo el mismo tipo de figura contractual. Hay diferencias entre ambos sexos en cuanto al tipo de contrato (indefinido o temporal) cuestión que resulta fundamental para obtener una visión completa de la situación del mercado de trabajo desde una perspectiva de género. Dichos datos se analizan a continuación de forma exhaustiva.

Tabla 6: Distribución porcentual de la población asalariada por sexo, según tipo de contrato.

\begin{tabular}{|c|c|c|c|c|c|c|}
\hline \multirow{2}{*}{ AÑ } & \multicolumn{2}{|c|}{ TOTAL } & \multicolumn{2}{c|}{ INDEFINIDO } & \multicolumn{2}{c|}{ TEMPORAL } \\
\cline { 2 - 7 } & \% H & \% M & \% H & \% M & \% H & \% M \\
\hline 1T 2007 & 57,1 & 42,9 & 57,9 & 42,1 & 55,3 & 44,7 \\
\hline 2T 2007 & 56,9 & 43,1 & 57,8 & 42,2 & 55,0 & 45,0 \\
\hline 3T 2007 & 56,9 & 43,1 & 57,7 & 42,3 & 55,1 & 44,9 \\
\hline 4T 2007 & 56,4 & 43,6 & 57,5 & 42,5 & 53,9 & 46,1 \\
\hline 1T 2008 & 56,2 & 43,8 & 57,4 & 42,6 & 53,5 & 46,5 \\
\hline 2T 2008 & 55,8 & 44,2 & 57,0 & 43,0 & 53,1 & 46,9 \\
\hline 3T 2008 & 55,6 & 44,4 & 57,0 & 43,0 & 52,1 & 47,9 \\
\hline 4T 2008 & 54,9 & 45,1 & 56,4 & 43,6 & 50,9 & 49,1 \\
\hline 1T 2009 & 54,4 & 45,6 & 55,9 & 44,1 & 50,0 & 50,0 \\
\hline 2T 2009 & 54,3 & 45,7 & 55,4 & 44,6 & 51,2 & 48,8 \\
\hline 3T 2009 & 54,1 & 45,9 & 55,3 & 44,7 & 50,5 & 49,5 \\
\hline 4T 2009 & 53,9 & 46,1 & 54,7 & 45,3 & 51,3 & 48,7 \\
\hline 1T 2010 & 53,3 & 46,7 & 54,2 & 45,8 & 50,7 & 49,3 \\
\hline 2T 2010 & 53,6 & 46,4 & 54,3 & 45,7 & 51,5 & 48,5 \\
\hline
\end{tabular}

Fuente: Encuesta de Población Activa, Instituto Nacional de Estadística, Ministerio de Economía y Hacienda (http://www.ine.es/) y elaboración propia.

Resulta de interés analizar si durante la época de crisis se han detectado variaciones en las tendencias de contratación de hombres y mujeres. En este sentido se observa que, tal como REVESCO No 105 - MONOGRÁFICO: La economía social y la igualdad de género ISSN: 1885-8031 - www.ucm.es/info/revesco 
ya se ha comentado anteriormente para la población activa y la población ocupada, las mujeres han incrementado paulatinamente su presencia entre el colectivo de asalariados/as pasando de una representación del $42,9 \%$ al 46,4 \%, en contraposición a un menor peso de los varones. Esta misma tendencia se observa para la contratación indefinida y para la temporal. En la contratación indefinida el porcentaje de mujeres ha venido aumentando hasta crecer en más de tres puntos y medio porcentuales. En el caso de los contratos temporales ocurre lo mismo, en ellos las mujeres han incrementado su peso en alrededor de cuatro puntos porcentuales.

La evidencia empírica no hace otra cosa que poner de manifiesto la mayor participación laboral femenina que se ha venido registrando paulatinamente a lo largo de los últimos años. Sin embargo, a pesar de dicha tendencia, la evidencia empírica muestra que la proporción de mujeres entre la población contratada de forma indefinida es inferior al porcentaje de representación que mantienen entre la población asalariada. La situación opuesta se da en el caso de la contratación temporal: el porcentaje de mujeres excede el peso de éstas entre la población asalariada, lo cual significa que las mujeres se ven mayormente afectadas por la contratación temporal.

Tabla 7: Distribución porcentual de los trabajadores de la Economía Social por sexo, según tipo de relación.

\begin{tabular}{|c|c|c|c|c|c|c|}
\hline \multirow{2}{*}{ AÑ } & \multicolumn{2}{|c|}{ TOTAL } & \multicolumn{2}{c|}{ INDEFINIDA } & \multicolumn{2}{c|}{ TEMPORAL } \\
\cline { 2 - 7 } & \% H & \% M & \% H & \% M & \% H & \% M \\
\hline 1T 2007 & 59,2 & 40,8 & 60,6 & 39,4 & 56,6 & 43,4 \\
\hline 2T 2007 & 61,1 & 38,9 & 61,6 & 38,4 & 60,1 & 39,9 \\
\hline 3T 2007 & 60,9 & 39,1 & 61,1 & 38,9 & 60,5 & 39,5 \\
\hline 4T 2007 & 59,2 & 40,8 & 60,1 & 39,9 & 57,2 & 42,8 \\
\hline 1T 2008 & 58,1 & 41,9 & 60,1 & 39,9 & 54,0 & 46,0 \\
\hline 2T 2008 & 59,6 & 40,4 & 60,8 & 39,2 & 56,8 & 43,2 \\
\hline 3T 2008 & 59,0 & 41,0 & 60,0 & 40,0 & 56,5 & 43,5 \\
\hline 4T 2008 & 57,2 & 42,8 & 59,1 & 40,9 & 52,4 & 47,6 \\
\hline 1T 2009 & 55,9 & 44,1 & 58,9 & 41,1 & 48,5 & 51,5 \\
\hline 2T 2009 & 57,4 & 42,6 & 59,2 & 40,8 & 52,6 & 47,4 \\
\hline
\end{tabular}

REVESCO Nº 105 - MONOGRÁFICO: La economía social y la igualdad de género -

ISSN: 1885-8031 - www.ucm.es/info/revesco 


\begin{tabular}{|l|c|c|c|c|c|c|}
\hline 3T 2009 & 57,4 & 42,6 & 58,8 & 41,2 & 53,2 & 46,8 \\
\hline 4T 2009 & 55,9 & 44,1 & 58,0 & 42,0 & 50,0 & 50,0 \\
\hline 1T 2010 & 55,3 & 44,7 & 58,0 & 42,0 & 47,9 & 52,1 \\
\hline 2T 2010 & 56,6 & 43,4 & 58,3 & 41,7 & 51,8 & 48,2 \\
\hline
\end{tabular}

Fuente: Ministerio de Trabajo e inmigración. Dirección General de la Economía Social, del trabajo autónomo y de la responsabilidad social de las empresas (http://www.mtin.es).

En el sector de la Economía Social se pueden observar cifras y tendencias similares a las registradas para el conjunto de la población asalariada. El peso de las mujeres en el sector se ha visto incrementado paulatinamente (como ya se ha comentado anteriormente) y éste es superior al peso del colectivo femenino entre el grupo contratado de forma indefinida y a la vez es inferior al peso que representan las mujeres en el colectivo contratado de forma temporal. Estos resultados, por tanto, indican que en el sector de la Economía Social también las mujeres acusan de forma más intensa la situación de precariedad laboral que supone la contratación de carácter temporal.

Gráfico 1: Distribución porcentual de la población asalariada por tipo de contrato, según sexo.

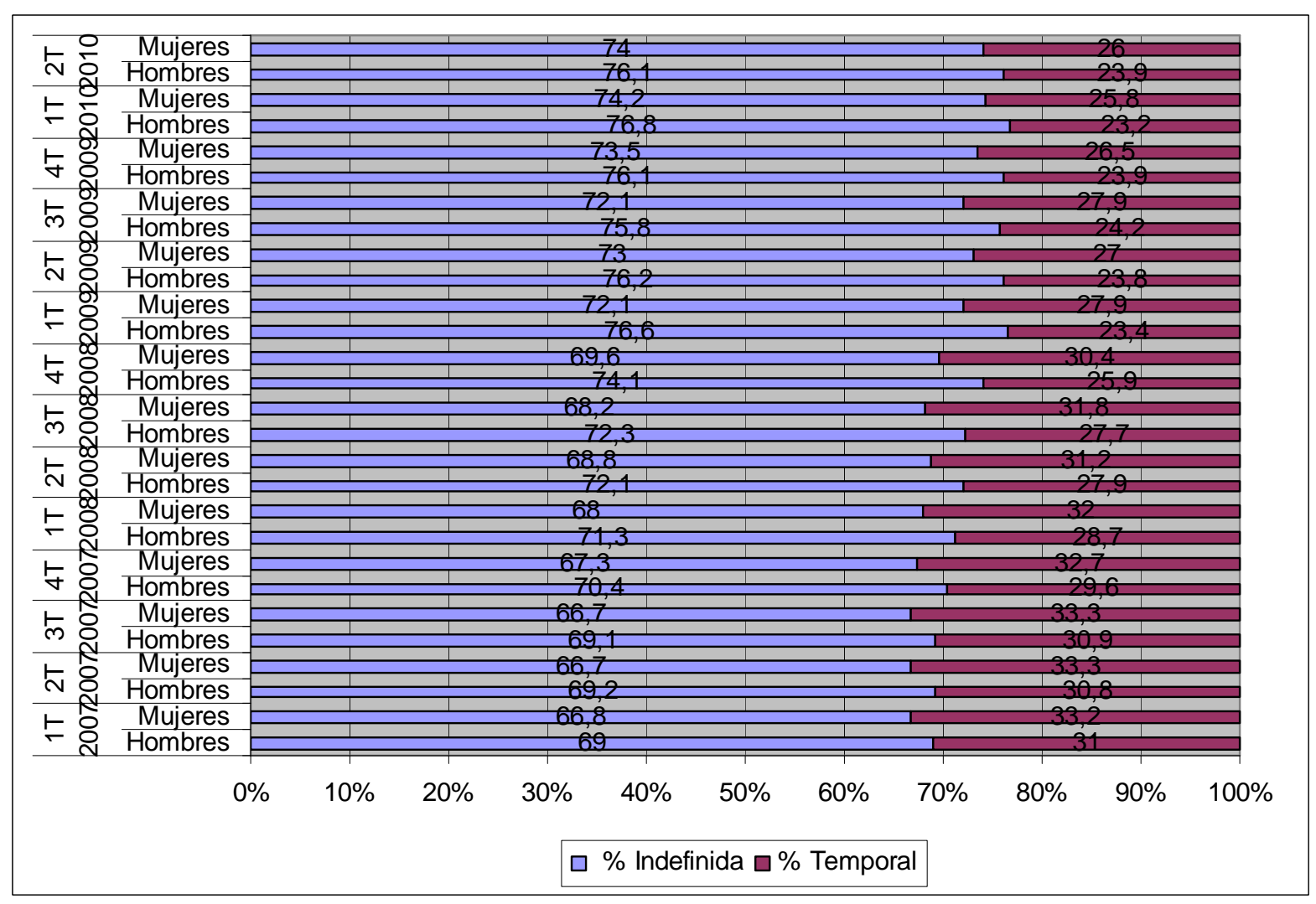

Fuente: Encuesta de Población Activa, Instituto Nacional de Estadística, Ministerio de Economía y Hacienda (http://www.ine.es/).

REVESCO Nº 105 - MONOGRÁFICO: La economía social y la igualdad de género -

ISSN: 1885-8031 - www.ucm.es/info/revesco 
A lo largo del período analizado se observa que entre los asalariados de la economía española, tanto hombres como mujeres, se produce un constante y significativo incremento de personas que están contratadas de forma indefinida en detrimento del porcentaje de personas (de uno y otro sexo) que están contratadas de forma temporal. A principios del 2007 un $69 \%$ de asalariados varones estaban contratados de forma indefinida mientras que a mitad del 2010 el porcentaje ha pasado a ser del 76,1 \%. En el caso de las mujeres asalariadas la tendencia es idéntica: al inicio del período un $66,8 \%$ de las mismas trabajaban con contratos indefinidos mientras que en el segundo trimestre del 2010 el porcentaje se ha incrementado hasta llegar al $74 \%$. No obstante lo anterior conviene resaltar que el porcentaje de mujeres asalariadas con contrato indefinido se mantiene dos puntos porcentuales por debajo del porcentaje de hombres que poseen dicho contrato. Este resultado pone de manifiesto una vez más la correlación existente entre colectivo femenino y contratación temporal.

Gráfico 2: Distribución porcentual de los trabajadores de la Economía Social por tipo de relación, según sexo.

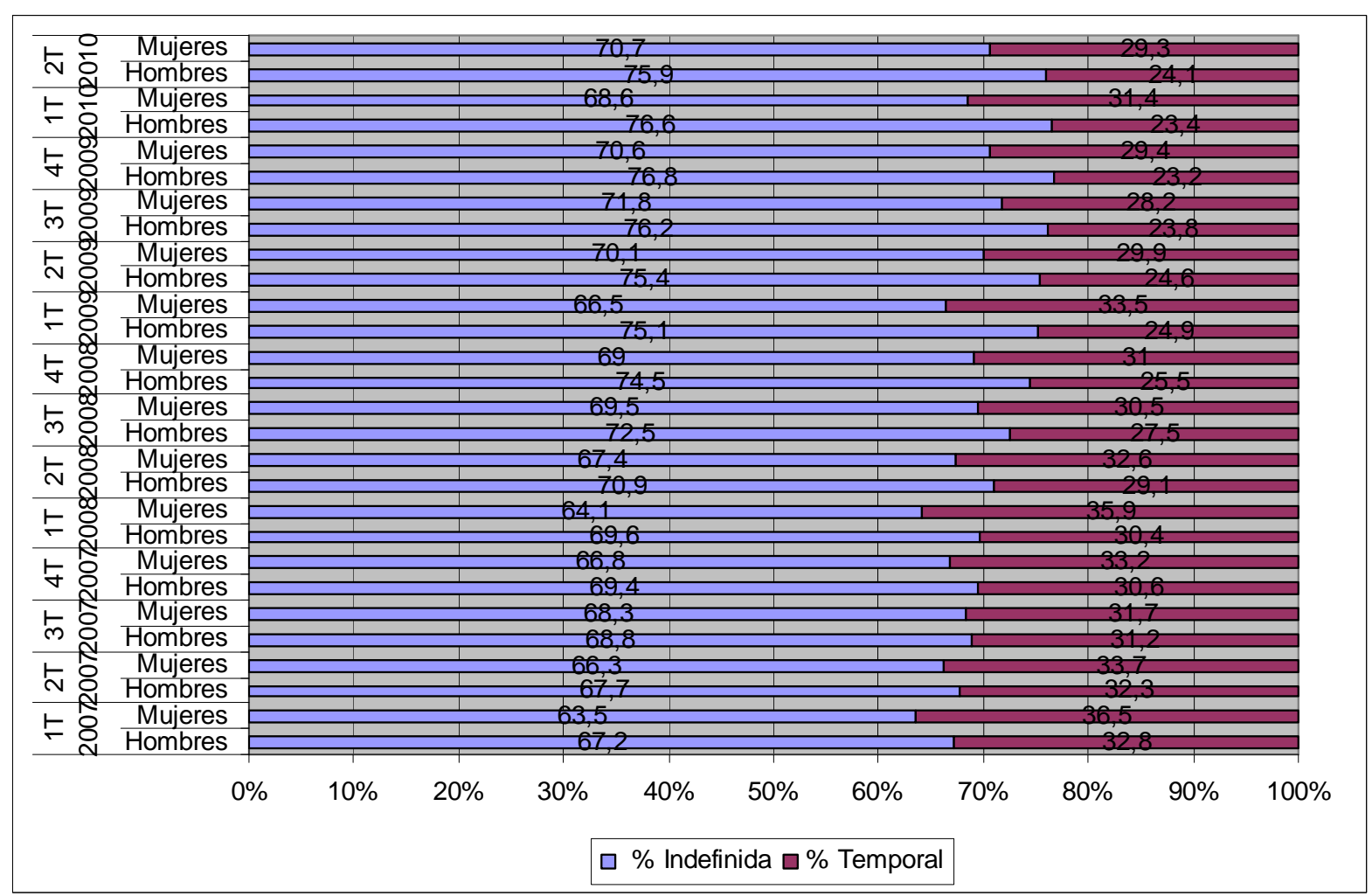

Fuente: Ministerio de Trabajo e inmigración. Dirección General de la Economía Social, del trabajo autónomo y de la responsabilidad social de las empresas (http://www.mtin.es).

REVESCO N 105 - MONOGRÁFICO: La economía social y la igualdad de género -

ISSN: 1885-8031 - www.ucm.es/info/revesco 
Los porcentajes y la tendencia de los trabajadores varones del sector de la Economía Social son similares a los registrados para el conjunto de los asalariados de la economía española, sin embargo, en el caso de las trabajadoras los resultados difieren. Aunque a lo largo del período se ve incrementada la proporción de mujeres contratadas de forma indefinida hasta llegar a un 70,7 \%, esta cifra es inferior a la registrada para las asalariadas a nivel estatal. Parece por tanto que entre el colectivo femenino de las entidades de Economía Social existe una mayor incidencia de contratación temporal que en el conjunto de la economía.

\section{COMPARACIÓN DEL TIPO DE JORNADA LABORAL EN HOMBRES Y MUJERES}

A continuación, y para cerrar el análisis desarrollado a la largo de este trabajo se presentan datos relativos al tipo de jornada laboral de hombres y mujeres. Al realizar un estudio empírico queda patente que los miembros de uno y otro sexo no dedican el mismo tiempo al trabajo remunerado, sino que existen importantes diferencias entre el porcentaje de varones y féminas que trabajan a tiempo parcial y a tiempo completo.

REVESCO Nº 105 - MONOGRÁFICO: La economía social y la igualdad de género -

ISSN: 1885-8031 - www.ucm.es/info/revesco 
Gráfico 3: Distribución porcentual de la población ocupada por tipo de jornada laboral, según sexo.

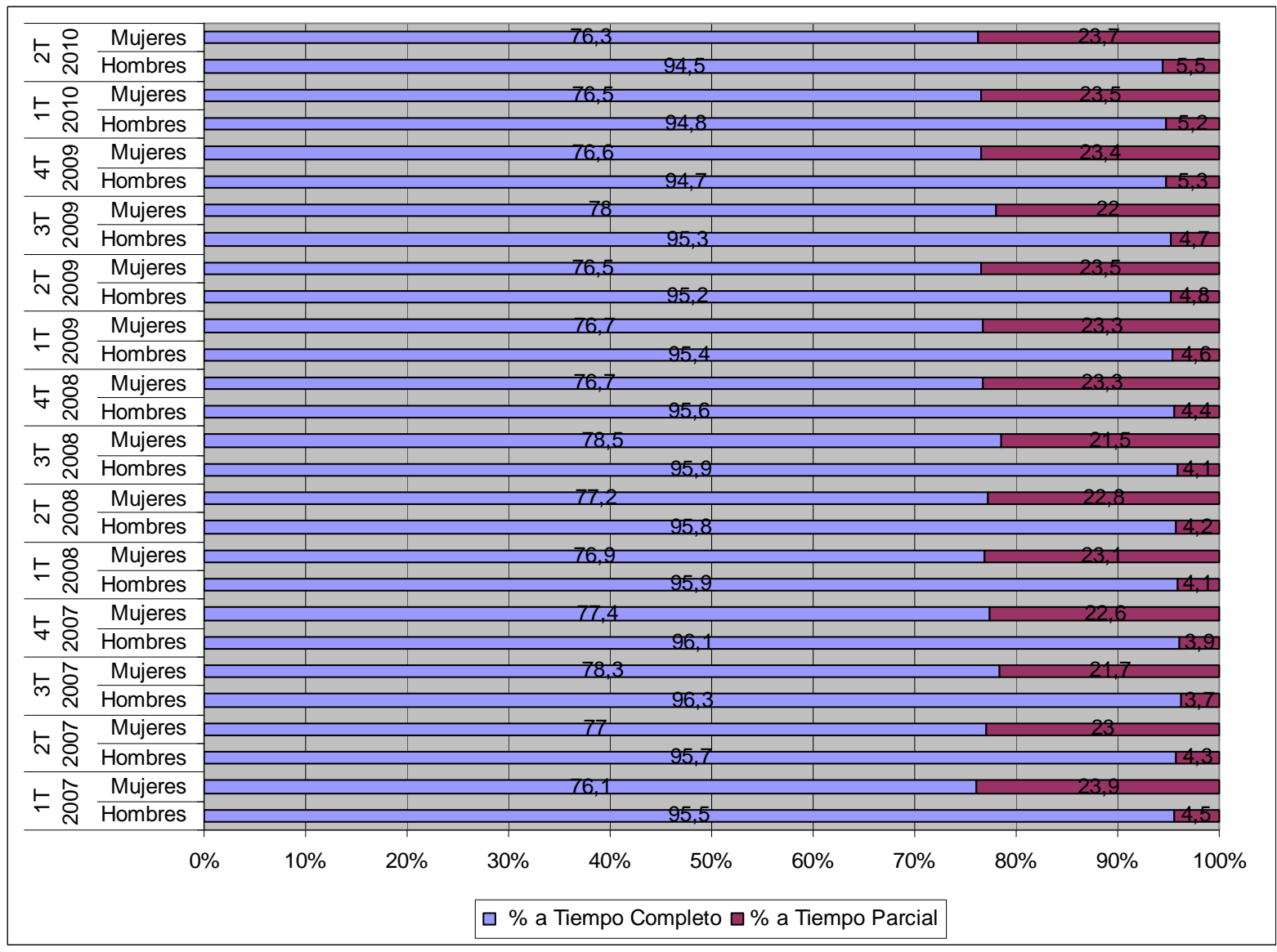

Fuente: Encuesta de Población Activa, Instituto Nacional de Estadística, Ministerio de Economía y Hacienda (http://www.ine.es/).

En la economía española los hombres trabajan mayoritariamente a tiempo completo siendo el porcentaje de trabajadores que lo hace a tiempo parcial meramente residual llegando al 5,5\% en el segundo trimestre del 2010, mientras que el porcentaje de mujeres que trabaja a tiempo parcial llega al $23,7 \%$ en dicho período. Al analizar en mayor profundidad estos resultados, las estadísticas del INE (Instituto Nacional de Estadística: http://www.ine.es/) indican que el trabajo a jornada parcial en el caso de los hombres viene principalmente motivado por no haber podido conseguir trabajo a tiempo completo o por motivos de enfermedad o incapacidad. Por el contrario, en el caso de las mujeres, aparte del hecho de no haber podido conseguir un empleo a jornada completa, tienen un mayor peso los motivos familiares como son el cuidado de niños, mayores o enfermos, así como la decisión personal de no querer trabajar a jornada completa.

REVESCO Nº 105 - MONOGRÁFICO: La economía social y la igualdad de género -

ISSN: 1885-8031 - www.ucm.es/info/revesco 
Gráfico 4: Distribución porcentual de los trabajadores de la Economía Social por tipo de jornada laboral, según sexo.

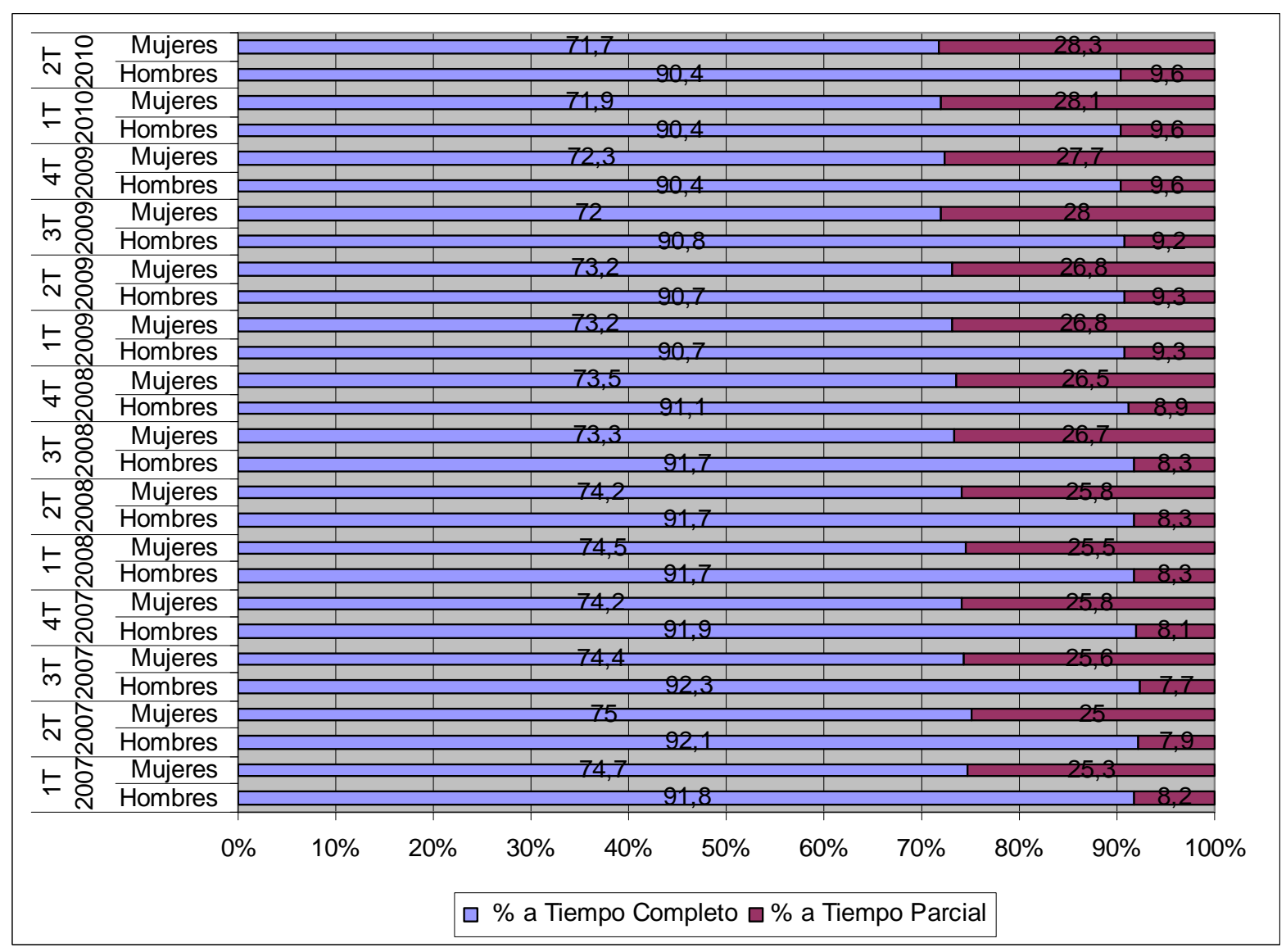

Fuente: Ministerio de Trabajo e inmigración. Dirección General de la Economía Social, del trabajo autónomo y de la responsabilidad social de las empresas (http://www.mtin.es).

En el sector de la Economía social también se observa la disparidad de tipología de jornada laboral registrada entre hombres y mujeres, presentado ésta una tendencia comparable a la detectada en el conjunto de la economía. No obstante, en dicho sector, el porcentaje de personas que trabajan a tiempo parcial es algo superior (unos cuatro puntos porcentuales) al porcentaje registrado por el INE para la población ocupada, y esto ocurre tanto en el caso de los hombres como en el caso de las mujeres, además, a lo largo el período se observa una ligera tendencia alcista en la proporción personas que trabajan a jornada parcial, llegando en el segundo trimestre del 2010 a un 9,6 \% en el caso de los hombres y un 28,3\% en el caso de las mujeres.

Las empresas de Economía Social, al ser empresas participativas facilitan una mayor implicación de los trabajadores, así como una mayor flexibilidad laboral, especialmente en 
épocas de crisis, que permite el ajuste de horarios, jornadas, turnos, etc. para adecuarse a las necesidades productivas del momento sin necesidad de pasar por la adopción de medidas de regulación de empleo. Este hecho podría ser uno de los factores que expliquen las diferencias entre los resultados obtenidos en el Sector de la Economía Social y en el conjunto de la economía en relación a la jornada laboral de hombres y mujeres.

\section{CONCLUSIONES}

La situación de crisis ha tenido efectos tanto en el colectivo masculino como en el femenino, aunque los datos indican que los hombres se han visto afectados en mayor medida por el desempleo y la disminución de la tasa de actividad, mientras que las mujeres han presentado un avance constante en su participación laboral y un incremento relativamente inferior de la tasa de paro.

En el sector de la Economía Social se observa una tendencia y unos datos similares de participación de hombres y mujeres a los registrados en el conjunto de la economía, con la salvedad que debe realizarse de la menor participación femenina en las sociedades laborales (anónimas y limitadas) en relación a las cooperativas.

Al igual que en el resto de la economía española, en las empresas de la Economía Social se observan diferencias de participación según los sectores. El dato más significativo es la mayor presencia femenina en el sector agrícola que supera en más de 20 puntos porcentuales a la registrada entre el conjunto de la población ocupada.

A todos los niveles, tanto en el conjunto de la Economía como en el sector de la Economía Social se observa una mayor incidencia de la contratación temporal entre las mujeres. El peso del colectivo femenino entre la población contratada de forma temporal supera la proporción alcanzada por las mujeres entre los trabajadores del sector de la Economía Social, mientras se registra la situación inversa entre los contratados de forma indefinida: el porcentaje de mujeres con contrato indefinido es inferior al peso de las mujeres en el sector. El porcentaje de mujeres con contrato indefinido es inferior al porcentaje de hombres con dicho contrato. Además, la cifra de mujeres contratadas de forma indefinida en 
el sector de la Economía Social es inferior a la registrada a nivel general para la economía española.

Se observa que las mujeres trabajan a tiempo parcial en mayor medida que los hombres, no obstante en el sector de la Economía Social se registra una mayor incidencia de este tipo de jornada tanto en el caso de los hombres como en el caso de las mujeres en relación a los datos observados para el conjunto de la economía.

\section{BIBLIOGRAFÍA}

INSTITUTO NACIONAL DE ESTADÍSTICA (INE): $\underline{\text { http://www.ine.es/. }}$ http://www.ine.es/jaxiBD/menu.do? $\mathrm{L}=0 \&$ divi=EPA\&his=1\&type=db

MINISTERIO DE TRABAJO E INMIGRACIÓN: http://www.mtin.es. http://www.mtin.es/es/sec trabajo/autonomos/economiasoc/EconomiaSocial/estadisticas/index.htm

MINISTERIO DE TRABAJO E INMIGRACIÓN. Dirección general de la Economía Social, del trabajo autónomo y de la responsabilidad social de las empresas: http://www.mtin.es/es/sec trabajo/autonomos/economiasoc/EconomiaSocial/estadisticas/CaracteristicasTrabajadores/index.htm

RIBAS BONET, Ma Antonia. Mujer y trabajo en la Economía Social. Madrid: Consejo Económico y Social, 2005, 283 p., ISBN: 84-8188-252-6. Prólogo de Baleren Bakaikoa Azurmendi

RIBAS BONET, M ${ }^{\mathrm{a}}$ Antonia. Mujer y trabajo en la Economía Social. Palma de Mallorca: Edicions UIB, 2010, 368 p., ISBN: 978-84-8384-148-8. Prólogos de Baleren Bakaikoa Azurmendi y de Inmaculada Serra Yoldi.

RIBAS BONET, Ma Antonia; SAJARDO MORENO, Antonia. La desigual participación de hombres y mujeres en la Economía Social: teorías explicativas. CIRIEC-España, Revista de la Economía Social, Pública y Cooperativa, № 50, 2004, p. 77-103.

RIBAS BONET, $M^{a}$ Antonia; SAJARDO MORENO, Antonia. La diferente participación laboral de las mujeres entre las cooperativas y las sociedades laborales. CIRIEC-España, Revista de la Economía Social, Pública y Cooperativa, No 52, 2005, p. 267-278.

REVESCO No 105 - MONOGRÁFICO: La economía social y la igualdad de género -

ISSN: $1885-8031$ - www.ucm.es/info/revesco 
SENENT VIDAL, $\mathrm{M}^{\mathrm{a}}$ José. Principios cooperativos, equidad de género y gobierno corporativo. 27 Congreso Internacional CIRIEC sobre Economía Pública, Social y Cooperativa, Sevilla, del 22 al 24 de septiembre de 2008.

REVESCO Nº 105 - MONOGRÁFICO: La economía social y la igualdad de género -

ISSN: 1885-8031 - www.ucm.es/info/revesco 